\title{
Die Massenstabilität des Elektrons ${ }^{1}$
}

\author{
Von Fritz Bopp \\ Aus dem Kaiser-Wilhelm-Institut für Physik, Hechingen \\ (Z. Naturforschg. 1, 53-58 [1946]; eingegangen am 24. Oktober 1945)
}

\begin{abstract}
Es werden Mindestbedingungen entwickelt, denen eine von der $\mathrm{M} \mathrm{ax} \mathrm{w} \mathrm{e} \mathrm{ll} \mathrm{schen} \mathrm{Theorie}$ abweichende Elektrodynamik genügen muß, um mit grundlegenden Erfahrungen verträglich zu sein. Insbesondere wird die Bedingung der Massenstabilität untersucht, die in einer Reihe bisher diskutierter Ansätze für die Feldtheorie (von Dirac, Stueckelberg u.d. Verf.) nicht gewahrt ist. Diese Bedingung besagt, daß die mit dem Felde variierende Masse des Elektrons nach Wechselwirkung mit äußeren Kräften wieder ihrem Gleichgewichtswert zustreben soll. Zum Schluß werden verschiedene in der Literatur diskutierten Ansätze zur Abänderung der $\mathrm{M}$ a x w e $1 \mathrm{l}$ schen Gleichungen im Lichte der formulierten Bedingungen verglichen und um einen ergänzt, der sämtlichen Bedingungen genügt.
\end{abstract}

W. Wessel ${ }^{2}$ und P.A. M. Dirac ${ }^{3}$ haben erstmalig auf eine bemerkenswerte Schwierigkeit hingewiesen, die sich ergibt, wenn man die Strahlungskraft und, wie man vom Standpunkt der allgemeinen Feldmechanik hinzufügen muß, auch die Trägheitskraft nicht nur als Näherung, sondern als strengen Ausdruck für Strahlungs- und Trägheitsrückwirkung betrachtet. In nichtrelativistischer Näherung lauten die Bewegungsgleichungen ${ }^{4}$

$$
\mathrm{m} \dot{\mathfrak{v}}-\frac{2}{3} \frac{\mathrm{e}^{\mathfrak{3}}}{\mathrm{c}^{3}} \ddot{\mathfrak{v}}=\Re \text {. }
$$

Diese kann man nach E.C.G.Stueckelberg ${ }^{5}$ auf folgende für die weitere Diskussion wichtige Form bringen:

$$
m \dot{\mathfrak{v}}=\int N\left(t-t^{\prime}\right) \Re\left(t^{\prime}\right) d t^{\prime} .
$$

Sie nehmen so die geläufige Form einer Bewegungsgleichung an. Nur erscheint die Kraft durch eine lineare Integraltransformation modifiziert. Die modifizierende Funktion ist durch das Integral

$$
N(t)=\frac{1}{2 \pi i} \int \frac{e^{p t} d p}{M(p)}=\frac{1}{2 \pi i} \int \frac{e^{p t} d p}{1-\lambda_{0} p}
$$

gegeben $\left(\right.$ mit $\left.\lambda_{0}=2 \mathrm{e}^{2} / 3 \mathrm{mc}^{3}\right)$, dessen Wert von dem Verlauf des Integrationsweges abhängt. Dirac und Stueckelberg bestimmen diesen so, daß

1 Auszug einiger neuer Ergebnisse aus einem zusammenfassenden Bericht über „Felder und Teilchen“, der in der Physikalischen Zeitschrift erscheinen sollte (Einsendedatum 16. Dez. 1944, Datum der Korrektur 2. März 1945).

2 Ann. Physik [5] 43, 565 [1943].
$\mathrm{N}(\mathrm{t})=0$ ist für alle Zeiten $t \geq 0$. Die modifizierte Kraft wirkt also in diesem Falle, bevor die eigentliche Kraft einsetzt. Diese überraschende, dem Kausalitätsprinzip widersprechende Festsetzung wird damit begründet, daß sich bei anderer Wahl ein sehr rasch exponentiell anwachsender Ausdruck für die modifizierte Kraft ergibt, die nach Wechselwirkung mit einem äußeren Kraftfeld zur ständigen Beschleunigung des Elektrons führt.

Wir haben bereits früher betont ${ }^{8}$, daß die Durchbrechung des Kausalitätsprinzips innerhalb der klassischen Feldtheorie nicht sehr zwingend erscheint, und daß man die Frage eher umgekehrt stellen sollte. Wie muß eine Feldtheorie aussehen, damit die oben geschilderte Schwierigkeit nicht auftritt? Schon Dirac hat auf diese Alternative hingewiesen, ohne ihre Diskussion zu erwägen. In der zitierten Arbeit haben wir bereits einige Gesichtspunkte zur Beantwortung dieser Frage beigetragen (im wesentlichen durch Untersuchung der kräftefreien Bewegung). Die Stueckelberg sche Form der Bewegungsgleichungen in Gl. (2) und Gl. (3) macht eine endgültige und präzise Formulierung möglich.

Die modifizierende Funktion $\mathrm{N}(\mathrm{t})$ soll eine reine Nachwirkungsfunktion sein, also für $t<0$ ver-

3 Proc. Roy. Soc. [London] Ser. A 167, 148 [1938].

4 Vergl. z. B. R. B e c k e r, Theorie der Elektrizität II, Berlin 1933, S. 77.

5 Helv. physica Acta 17, 3 [1944].

- F. B op p, Ann. Physik [5] 42, 572 [1943]. 
schwinden und keine exponentiell anwachsenden Glieder enthalten. Die erste Forderung besagt, daß der Integrationsweg in Gl. (3) alle Singularitäten links liegen läßt. Die zweite Forderung ist erfüllt, wenn die Funktion $M(p)$ im Nenner des Integranden in Gl. (3) keine Nullstellen oder Verzweigungspunkte in der rechten Halbebene hat.

Die anschauliche Bedeutung der Nachwirkungsfunktion ist leicht zu erkennen. In der Feldmechanik sind Trägheits- und Strahlungskraft zunächst Funktionen des Eigenfeldes und erst dadurch von Geschwindigkeit, Beschleunigung und den höheren Beschleunigungen des Teilchens abhängig. Der spezielle Ausdruck für die Masse $m=m_{0} / \sqrt{1-\beta^{2}}$ ist im allgemeinen nur für die geradlinige, gleichförmige Bewegung möglich und auch da keineswegs notwendig. Das Feld eines unter dem Einfluß einer äußeren Kraft beschleunigten Teilchens wird deformiert, was zu einer Änderung der Selbstenergie und Masse führt. Nach dem Aufhören der Wechselwirkung mit der äußeren Kraft stellt sich nicht sofort das gewöhnliche Feld des gleichförmig und geradlinig bewegten Teilchens ein. Der Übergang vollzieht sich vielmehr, wenn er überhaupt möglich ist, mit einer gewissen Trägheit, die zu einer Nachwirkung der Kraft führt. Die Nachwirkungsfunktion in Gleichung (2) beschreibt also eine Art Relaxationserscheinung.

Im allgemeinen ist jedoch, wie Beispiele von Dirac ${ }^{3}$, Stueckelberg ${ }^{5}$ und B op $p^{6}$ zeigen, die Rückkehr zur Gleichgewichtsmasse überhaupt nicht möglich. Das einmal aus der geradlinig und gleichförmig durchlaufenen Bahn herausgerissene Teilchen kann nicht mehr in diese zurückkehren, weil die Bewegung am Anfang offenbar einen labilen Zustand darstellt. Da der Energiesatz gilt, kann die Beschleunigung des Diracschen Elektrons nur auf Kosten einer ständig schwindenden Masse erfolgen, um so mehr als die Beschleunigung noch von Ausstrahlung begleitet ist. Unsere Forderung, daß die Funktion $\mathrm{M}(\mathrm{p})$ in der positiven Halbebene weder Nullstellen noch Verzweigungspunkte haben

7 G. Mi e, Ann. Physik [4] 37, 511 [1912]; M. B or n, Proc. Roy. Soc. [London] Ser. A 143, 410 [1933]; vergl. 1.c. ${ }^{8}$.

8 F. B op p, Ann. Physik [5] 38, 549 [1940].

9 Das Integrationselement lautet: $\mathrm{d} \mathbf{x}=\mathrm{d} \tau \mathrm{d} t$; entsprechend setzen wir später: $\mathrm{d} \mathbf{k}=\mathrm{d} \tau_{\mathrm{p}} \mathrm{d} \omega$.

10 Auf Grund des Hilbertschen Algorithmus wurde dieser in einer Arbeit abgeleitet, die im Juli 1944 an Ann. Physik gesandt wurde und die wir noch an dieser Stelle auszugsweise bekannt machen werden. darf, erscheint hiernach als Bedingung zur Sicherstellung der Massenstabilität.

Im folgenden handelt es sich darum, die Auswirkung dieser Bedingung der Massenstabilität auf den Ansatz der Feldtheorie zu diskutieren. Die einfache von Dirac angegebene Verallgemeinerung der Maxwellschen Theorie, (die dem üblichen Verfahren entspricht, außerhalb der Singularität Coulombsche Wechselwirkung anzunehmen, gleichwohl aber mit endlicher Masse zu rechnen), genügt jedenfalls nicht dieser Bedingung. Leider ist die charakteristische Funktion $M(p)$ nicht von solcher Art, daß sich damit alle Anforderungen, denen eine der Wirklichkeit sich annähernde Feldtheorie genügen muß, in einfacher Weise beschreiben lassen. Um einen gemeinsamen Bezugspunkt für die verschiedenen Bedingungen zu finden, beginnen wir mit einem allgemeinen Ansatz für die Feldgleichungen, der zunächst jede beliebige Modifikation des Coul o mb-Feldes zuläßt.

Die Feldgleichungen haben wie in der MieB ornschen Theorie ${ }^{7}$ die von den M axwellschen Gleichungen für substanzielle Medien geläufige Form

$$
\frac{\partial \mathrm{F}_{\mu \nu}}{\partial \mathrm{x}_{\nu}}=\frac{4 \pi}{\mathrm{c}} \mathrm{s}_{\mu}, \mathrm{f}_{\mu \nu}=\frac{\partial \varphi_{\nu}}{\partial \mathrm{x}_{\mu}}-\frac{\partial \varphi_{\mu}}{\partial \mathrm{x}_{\nu}} .
$$

Der Zusammenhang zwischen der Felderregung F und der Feldstärke $\mathrm{f}$ wird jedoch linear angenommen $^{8}$ :

$$
\left.\mathrm{F}_{\mu \nu}(\mathrm{x})=\int \varepsilon\left(\mathrm{x}-\mathrm{x}^{\prime}\right) \mathrm{f}_{\mu \nu}\left(\mathrm{x}^{\prime}\right) \mathrm{d} \mathrm{x}^{\prime}=\varepsilon, \mathrm{f}_{\mu \nu}(\mathrm{x})^{9}\right) .
$$

Darin darf die Funktion $\varepsilon(\mathrm{x})$ wegen der Lorentz-Invarianz nur von $\tau=\sqrt{-\mathrm{x}_{\mu^{2}}}$ abhängen. Der Energie-Impuls-Satz hat die gewöhnliche, leicht $\mathrm{zu}$ verifizierende Form:

$$
\frac{\partial \mathrm{T}_{\mu \nu}}{\partial \mathrm{x}_{v}}=-\frac{1}{\mathrm{c}} \mathrm{f}_{\mu \nu} \mathrm{s}_{v} .
$$

Statt den Energie-Impuls-Tensor selbst anzugeben $^{10}$, schreiben wir im Anschluß an W.Heisenber ${ }^{11}$ seine F ourier-Komponenten ${ }^{12}$ an:

11 Theorie der Elementarteilchen III, Z. Physik, zum Druck eingereicht. Für die Überlassung der Niederschrift vor der Veröffentlichung danke ich herzlich.

12 Die Fourier-Komponenten wollen wir mit denselben Buchstaben bezeichnen wie die zugehörigen Funktionen und durch eine Tilde über den Buchstaben von diesen unterscheiden gemäß:

$$
\begin{gathered}
f(x)=\frac{1}{16 \pi^{4}} \int \tilde{f}(k) e^{i k \mu x} \mu d k \\
\tilde{f}(k)=\int \varepsilon(x) e^{-i k \mu x} \mu d x .
\end{gathered}
$$




$$
\begin{aligned}
\tilde{\mathrm{T}}_{\mu \nu}(\mathbf{k}) & =\frac{1}{128 \pi^{j}} \int\left(\mathrm{k}_{\mu}-2 \mathrm{k}_{\mu}^{\prime}\right)\left(\mathrm{k}_{\nu}-2 \mathrm{k}^{\prime}{ }_{\nu}\right) \frac{\left(\mathrm{k}-\mathrm{k}^{\prime}\right)^{2} \tilde{\varepsilon}\left(\mathrm{k}-\mathrm{k}^{\prime}\right)-\mathrm{k}^{\prime 2} \tilde{\varepsilon}\left(\mathrm{k}^{\prime}\right)}{\left(\mathrm{k}-\mathrm{k}^{\prime}\right)^{2}-\tilde{\varphi}_{Q}\left(\mathrm{k}^{\prime 2}\right) \tilde{\varphi}_{Q}\left(\mathrm{k}-\mathrm{k}^{\prime}\right) \mathrm{d} \mathrm{k}^{\prime}} \\
& -\frac{1}{32 \pi^{4} \mathrm{c}} \delta_{\mu \nu} \int \tilde{\varphi}_{\underline{Q}}\left(\mathrm{k}^{\prime}\right) \tilde{\mathrm{s}}_{\varrho}\left(\mathrm{k}-\mathrm{k}^{\prime}\right) \mathrm{d} \mathrm{k}^{\prime}+\frac{1}{16 \pi^{4} \mathrm{c}} \int \tilde{\varphi}_{\mu}\left(\mathrm{k}^{\prime}\right) \tilde{\mathrm{s}}_{\nu}\left(\mathrm{k}-\mathrm{k}^{\prime}\right) \mathrm{d} \mathrm{k}^{\prime} .
\end{aligned}
$$

In Übereinstimmung mit Gl. (6) verifiziert man leicht die Gleichung

$$
\mathrm{i}_{\nu} \tilde{\mathrm{T}}_{\mu \nu}=-\frac{1}{16 \pi^{4} \mathrm{c}} \int\left[\mathrm{k}_{\mu}^{\prime} \tilde{\mathscr{T}}_{\nu}\left(\mathrm{k}^{\prime}\right)-\mathrm{k}_{\nu}^{\prime} \tilde{\varphi}_{\mu}\left(\mathrm{k}^{\prime}\right)\right] \tilde{\mathrm{s}}_{\nu}\left(\mathrm{k}-\mathrm{k}^{\prime}\right) \mathrm{d} \mathrm{\textrm {k } ^ { \prime }}
$$

Diese Gleichungen mögen auf zwei wichtige Spezialfälle angewandt werden. Im ladungsfreien Falle führen die Feldgleichungen zu den homogenen Wellengleichungen

$$
\square\left(\varepsilon, \varphi_{\mu}\right)=0, \quad \mathrm{k}_{\nu}^{2} \tilde{\varepsilon}\left(\mathrm{k}^{2}\right) \tilde{\varphi}_{\mu}(\mathrm{k})=0
$$

Die Komponenten $\tilde{\varphi}_{\mu}(\mathrm{k})$ können nur an solchen Stellen von Null verschieden sein, für die $\mathrm{k}^{2} \tilde{\varepsilon}\left(\mathrm{k}^{2}\right)$ $=0$ ist, d. h. für

$$
\mathrm{k}_{v}^{02}=0, \quad \mathrm{k}_{v}^{02}=-\varkappa^{2},
$$

wobei $-x^{2}$ die Nullstellen der Funktion $\tilde{\varepsilon}\left(k^{2}\right)$ durchläuft. Man erhält also als homogene Wellen Photonen und eventuell, entsprechend den Nullstellen von $\varepsilon\left(\mathrm{k}^{2}\right)$, eine Anzahl von neutralen Mesonen verschiedener Masse. Für die Energiedichte ergeben sich die im Anschluß an Gl. (7) leicht zu verifizierenden Ausdrücke

für die Photonen und

$$
\mathrm{W}=\begin{array}{ccc}
1 & \omega_{0}^{2} & \\
8 \pi & \mathrm{c}^{2} & \mathrm{~A}_{\underline{Q}}^{2}
\end{array}
$$

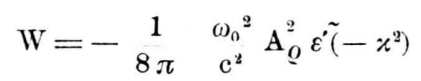

für die Mesonen. Der erste Ausdruck ist von der speziellen Feldtheorie unabhängig und stimmt darum mit dem geläufigen Ausdruck für $\mathrm{Max}$ wellsche Photonen überein. Der zweite kann je nach dem Vorzeichen von $\tilde{\varepsilon}^{\prime}$ positiv und negativ sein. Der von B op p ${ }^{8}$ und A. Land $\dot{e}^{13}$ diskutierte Ansatz $\tilde{\varepsilon}(\mathrm{k})=1+\mathrm{k}_{\mu}^{2} / \mathrm{x}^{2}$ liefert $\mathrm{z}$. B. negative Quanten.

Für das elektrostatische Potential einer ruhenden Punktladung (modifiziertes Coulomb-Potential) erhält man aus

$$
\triangle(\varepsilon, \varphi)=-4 \pi \text { e } \delta(\mathfrak{r}), \quad \mathrm{k}_{\mu}^{2} \tilde{\varepsilon}(\mathrm{k}) \tilde{\varphi}(\mathrm{k})=4 \pi \mathrm{e}
$$

die Gleichung

$$
\varphi(\mathrm{r})=\frac{2}{\pi} \frac{\mathrm{e}}{\mathrm{r}} \int_{0}^{\infty} \frac{\sin \mathrm{kr}}{\mathrm{k} \tilde{\varepsilon}(\mathfrak{f}, \mathrm{o})} \mathrm{dk},
$$

13 Physic. Rev. 60, 121 [1941]; A. Landé u. L. H. Th o mas, Physic. Rev. 60, 514 [1941]. deren Auflösung nach $\tilde{\varepsilon}$ folgendermaßen lautet:

$$
\frac{1}{\tilde{\varepsilon}(\mathfrak{k}, \mathrm{o})}=-\frac{1}{\mathrm{e}} \mathrm{k} \frac{\mathrm{d}}{\mathrm{d} \mathrm{k}} \int \varphi(\mathrm{r}) \cos \mathrm{kr} \mathrm{d} \mathbf{r} .
$$

$\mathrm{Zu}$ jedem Potentialansatz gibt es also mindestens einen Ansatz $\tilde{\varepsilon}$, der gerade das angenommene Potential liefert. Man kann zeigen - was hier nur erwähnt sei -, daß es auch nur einen Ausdruck $\tilde{\varepsilon}$ gibt, der das leistet, wenn man im Sinne des Kausalitätsprinzips fordert, daß sich das Viererpotential retardiert aus der Ladungs- und Stromverteilung berechnet. Für die Energie der ruhenden Punktladung erhält man aus

$\mathrm{E}=-\int \mathrm{T}_{44}(r, \mathrm{t}) \mathrm{d} \tau=-{ }_{2 \pi}^{1} \int \tilde{\mathrm{T}}_{44}(o, \omega) \mathrm{e}^{-\mathrm{i} \omega \mathrm{t}} \mathrm{d} \omega$

mit Rücksicht auf Gl. (7)

$$
\mathrm{E}_{0}=\frac{1}{2} \mathrm{e} \varphi(\mathrm{o}) \text {. }
$$

Ehe wir zur Formulierung der Bedingung der Massenstabilität zurückkehren, müssen wir noch die Bewegungsgleichungen angeben. Wir können uns im Anschluß an eine frühere Arbeit kurz fassen $^{6}$. Das Viererpotential hat die in einem speziellen Fall auf D ir a c zurückgehende Form

$$
\varphi_{\mu}=\frac{2}{\mathrm{c}^{2}} \int \mathrm{g}\left(\mathrm{x}-\mathrm{x}^{\prime}\right) \mathrm{s}_{\mu}\left(\mathrm{x}^{\prime}\right) \mathrm{d} \mathrm{x}^{\prime} .
$$

Darin ist $\mathrm{g}$ durch das Integral

$$
g(\mathbf{x})=\frac{\mathrm{c}}{8 \pi^{3}} \int_{\text {ret }} \frac{\mathrm{e}^{\mathrm{i} \mathrm{k} \mu^{\mathrm{x}} \mu}}{\mathrm{k}_{Q}^{2} \tilde{\varepsilon}(\mathrm{k})} \mathrm{dk}
$$

bestimmt, wobei der Integrationsweg ",retardiert" $\mathrm{zu}$ wählen ist, d. h. so, daß $\mathrm{g}$ für alle Zeiten $\mathrm{t}<0$ verschwindet. Aus Gründen der Lor en tz - Invarianz kann g nur von $\tau=\sqrt{-\mathrm{x}_{\mu}{ }^{2}}$ abhängen. Die Bewegungsgleichungen folgen aus der Forderung, dafs sich die Singularitäten im eigenen und fremden Felde so bewegen, wie es ohne zusätzliche Führungskräfte möglich ist. Der Ausdruck für die Kraft des Eigenfeldes (d.i. Trägheits- und Strahlungskraft) lautet: 


$$
\begin{aligned}
\Re_{\text {eigen }} & =\frac{2 \mathrm{e}^{\mathfrak{q}}}{\mathrm{c}^{3}} \int_{0}^{\infty}\left\{\tau \mathfrak{v}^{\prime}-\left(1-\frac{\mathfrak{v} \mathfrak{v}^{\prime}}{\mathrm{c}^{2}}\right)\left(\mathfrak{r}_{0}-\mathfrak{r}_{0}\right)-\right. \\
& \left.-\frac{1}{\mathrm{c}^{2}}\left(\mathfrak{v}, \mathfrak{r}_{0}-\mathfrak{r} \cdot{ }_{0}^{\prime}\right) \mathfrak{v}^{\prime}\right\} \frac{\mathrm{g}^{\prime}(\sigma)}{\sigma} \mathrm{d} \tau .
\end{aligned}
$$

Darin bedeutet $\mathrm{g}(\sigma)$ die oben angegebene Retardierungsfunktion, $\mathfrak{r}_{0}=\mathfrak{r}_{0}(t)$ und $\mathfrak{v}=\dot{\mathfrak{r}}_{0}(\mathrm{t})$ geben die Bahndaten zur Zeit $t, \mathfrak{r}_{0}{ }^{\prime}$ und $\mathfrak{v}^{\prime}=\dot{\mathfrak{r}}_{0}{ }^{\prime}$ dieselben zur Zeit $t^{\prime}=t-\tau$. In nicht relativistischer Näherung vereinfacht sich der Ausdruck zu

$\mathfrak{K}_{\text {eigen }}=\frac{2 \mathrm{e}^{2}}{\mathrm{c}^{3}} \int_{0}^{\infty}\left\{\mathfrak{v}(\mathrm{t}-\tau)-\frac{\mathfrak{r}_{0}(\mathrm{t})-\mathfrak{r}_{0}(\mathrm{t}-\tau)}{\tau}\right\} \mathrm{g}^{\prime}(\tau) \mathrm{d} \tau$.

Die rechte Seite stellt ein Integral über einen der Beschleunigung analogen Differenzenquotienten dar. Die Entwicklung der geschweiften Klammer nach der Retardierung ergibt nach einigen Umformungen eine Bewegungsgleichung der Form

$$
\mathrm{m}_{0} \mathrm{M}\left(\frac{\mathrm{d}}{\mathrm{d} \mathrm{t}}\right) \dot{\mathfrak{v}}=\mathfrak{R} .
$$

Darin ist $\mathrm{M}(\mathrm{p})$ durch die Reihe

$$
\mathbf{M}(\mathrm{p})=\frac{2 \mathrm{e}^{2}}{3 \mathrm{~m} \mathrm{c}^{\mathrm{s}}} \Sigma(-\mathrm{p})^{\mathrm{n}} \frac{(\mathrm{n}+1)^{2}}{(\mathrm{n}+2) !} \int_{0}^{\infty} \tau^{\mathrm{n}} \mathrm{g}(\tau) \mathrm{d} \tau
$$

definiert, die mit der Laplaceschen Transformierten $^{14} \stackrel{v}{g}$ von $g$ zusammenhängt. Es ist

$$
\mathrm{M}(\mathrm{p})=3 \lambda_{0}\left\{\mathrm{~g}(\mathrm{p})-\frac{1}{\mathrm{p}^{2}} \int_{0}^{\mathrm{p}} \mathrm{g}(\xi) \xi \mathrm{d} \xi\right\} .
$$

Die Nachwirkungsfunktion in Gl. (3) stellt eine Lösung der Differentialgleichung

$$
\mathrm{M}\left(\frac{\mathrm{d}}{\mathrm{dt}}\right) \mathrm{N}(\mathrm{t})=\delta(\mathrm{t})
$$

dar und führt Gl. (20) sofort in Gl. (2) über.

Damit ist der allgemeine Rahmen einer Feldtheorie entworfen, die sich sehr eng an die M axwellsche Theorie anschließt. Die Fülle der möglichen Ansätze wird durch eine Reihe von allgemeinen Grunderfahrungen eingeschränkt, die wir in folgenden fünf Bedingungen zusammenfassen:

${ }_{14} \mathrm{Laplacesche}$ Unterfunktionen und Oberfunktionen bezeichnen wir mit Akzenten gemäß:

$$
f(s)=\int^{\infty} \hat{f}(t) e^{-s t} d t, \stackrel{v}{g}(s)=\int_{0}^{\infty} g(t) e^{-s t} d t .
$$

I. Coulomb-Bedingung: Das statische Potential der ruhenden Punktladung ist weit draußen Coulombsch.

II. Endlichkeitsbedingung: Die Feldenergie der ruhenden Punktladung ist endlich und durch die Masse bestimmt.

III. Photonenbedingung: Die Feldtheorie läßt neben den Photonen keine weiterenQuanten zu.

IV. Strahlungsbedingung: Die Reaktionskräfte der Strahlung stimmen mit denen der Maxwellschen Theorie überein.

V. Bedingung der Massenstabilität: Nach Wechselwirkung mit einem äußeren Kraftfeld kehrt die Masse zu ihrem Gleichgewichtswert zurück.

Die erste Bedingung ist trivial. An der zweiten hat sich zum erstenmal die Notwendigkeit einer Abänderung der M axwellschen Theorie gezeigt. Die Strahlungsbedingung ist willkürlich und könnte durch spätere Erfahrungen noch abgeändert werden. Die Photonenbedingung geht auf Stueckelberg zurück. Sie schließt vor allem die Möglichkeit von Quanten negativer Energie aus. In der klassischen Theorie bereiten diese keine Schwierigkeiten, da die Photonenenergie die der negativen Quanten immer übersteigt. In der Quantentheorie könnten sich die negativen Quanten infolge von Schwankungserscheinungen durchsetzen $^{15}$. Die Stabilitätsbedingung tritt hier als wesentlich neu hinzu und ist bereits oben formuliert.

Wir haben verschiedene Funktionen kennen gelernt, von denen jede die speziell zu betrachtende Elektrodynamik kennzeichnen kann: $\varepsilon(\mathrm{x}), \tilde{\varepsilon}(\mathrm{k})$, $\varphi(r), M(p)$ und $g(x)$. Keine von ihnen gestattet aber sämtliche Bedingungen zugleich in durchsichtiger Weise zu formulieren. Dazu ist ein formaler Ausdruck besonders geeignet, dem keine besondere physikalische Bedeutung zukommt: die Potentialoberfunktion $\hat{\Phi}(\mathrm{s})$, aus der das elektrostatische Potential $\varphi(r)$ durch die folgende Laplace-Transformation hervorgeht:

$\varphi(\mathrm{r})=\frac{\Theta}{\lambda_{0} \mathrm{c}} \Phi\left(\frac{\mathrm{r}}{\lambda_{0} \mathrm{c}}\right) ; \quad \Phi(\varrho)=\int_{0}^{\infty} \hat{\Phi}(\sigma) \mathrm{e}^{-\sigma \varrho} \mathrm{d} \sigma$.

Speziell erhält man aus den beiden ersten Forderungen die beiden leicht zu verifizierenden Bedingungen :

15 Vergl. dazu A. L a n dé, Physic. Rev. 65, 175, [1944] 
Coulomb-Bedingung: $\quad \hat{\Phi}(0)=1 ;$

Endlichkeits-Bedingung: $\int_{0}^{\infty} \hat{\phi}(\sigma) \mathrm{d} \sigma=\frac{4}{3}$.

Die Photonenbedingung folgt aus Gl. (13), die nach Einführung der Oberfunktion folgende Form annimmt:

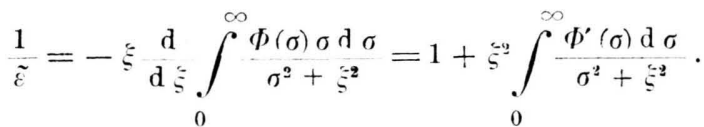

Darin ist $\quad \xi^{2}=\lambda_{0}^{2} \mathrm{c}^{2}\left({ }^{0} \mathfrak{k}^{2}-\frac{\omega^{2}}{\mathrm{c}^{2}}\right)$.

Wenn als Quanten nur Photonen zugelassen sind, darf $\tilde{\varepsilon}$ keine Nullstellen haben. Das Integral 1/ $\tilde{\varepsilon}$ muß also für alle Werte von $\xi$ endlich bleiben. Daraus folgt:

Photonenbedingung: $\hat{\phi}(\sigma)$ im ganzen Integrationsintervall stetig und stetig differenzierbar.

Eine Unstetigkeit, die $\hat{\Phi}(\sigma)$ verkleinert, liefert negative Quanten, eine, die $\hat{\Phi}(\sigma)$ vergrößert, positive. Die negativen Quanten werden durch die Stueckelbergsche Überlegung ausgeschlossen. Die positiven ziehen wir nicht in Betracht, weil sie einerseits die Erfüllung der Endlichkeitsbedingung erschweren, andrerseits durch zusätzliche Erfahrungen nicht gefordert werden.

Zur Untersuchung der Strahlungs- und Stabilitätsbedingung, bei denen wir etwas verweilen müssen, brauchen wir den Zusammenhang zwischen M (p) und $\hat{\phi}$ (s). Aus Gl. (16) erhält man für das statische Potential

$$
\Phi(\varrho)=2 \lambda_{0} \int_{0}^{\infty} \frac{g(t) d t}{\sqrt{t^{2}+\lambda_{0}^{2} \varrho^{2}}}
$$

und für die Oberfunktion

$$
\hat{\Phi}(\mathrm{s})=2 \int_{0}^{\infty} \mathrm{I}_{0}\left(\frac{\mathrm{s} t}{\lambda_{0}}\right) \mathrm{g}(\mathrm{t}) \mathrm{tdt} .
$$

Die Auflösung dieser Integralgleichung nach g ergibt

$$
g(t)=\frac{1}{2 \lambda_{0}^{2}} \int_{0}^{\infty} \mathrm{I}_{0}\left(\frac{\mathrm{ts}}{\lambda_{0}}\right) \hat{\phi}(\mathrm{s}) \mathrm{sds}
$$

und

$$
\mathrm{g}(\mathrm{p})=2 \lambda_{0}^{1} \int_{0}^{\infty} \frac{\hat{\phi}(\mathrm{s}) \mathrm{s} d \mathrm{~s}}{\sqrt{\mathrm{p}^{2}+\mathrm{s}^{2} / \lambda_{0}^{2}}}
$$

und nach Gl. (22) schließlich

$$
\mathrm{M}\left(\frac{\mathrm{x}}{\lambda_{0}}\right)=\frac{3}{2 \mathrm{x}^{2}} \int_{0}^{\infty}\left(1-\frac{\mathrm{s}}{\sqrt{\mathrm{s}^{2}+\mathrm{x}^{2}}}\right) \hat{\phi}(\mathrm{s}) \mathrm{s}^{2} \mathrm{~d} \mathrm{~s} .
$$

Zunächst ziehen wir aus der letzten Gleichung eine Folgerung für die Strahlungskraft. Nach $\mathrm{Bopp}{ }^{9}$ ist diese durch die ungeraden Potenzen einer Entwicklung von $M(p)$ nach $p$ gegeben. Das lineare Glied, nämlich $-\mathrm{x}$, liefert speziell den geläufigen Ausdruck für die Strahlungskraft. Nach Bedingung IV dürfen also höhere ungerade Potenzen nicht vorkommen. Setzen wir

$\mathrm{M}\left(\frac{\mathrm{x}}{\lambda_{0}}\right)=\frac{3}{2}\left(\frac{\mathrm{f}(\mathrm{x})}{\mathrm{x}}\right)^{\prime}, \mathrm{f}(\mathrm{x})=\int_{0}^{\infty}\left(\sqrt{\left.\mathrm{s}^{\mathrm{x}}+\mathrm{x}^{2}-\mathrm{s}\right) \hat{\phi}(\mathrm{s}) \mathrm{s} \mathrm{d} \mathrm{s},}\right.$

so führt eine partielle Integration zu dem Ausdruck

$f(x)=-\frac{1}{3} x^{3}-\frac{1}{3} \int_{0}^{\infty}\left\{\sqrt{s^{2}+x^{2}}-s^{3}\right\} \hat{\phi}^{\prime}(s) d s$.

Das erste Glied liefert den gew ünschten, linearen Beitrag. Weitere ungerade Potenzen fallen weg, wenn wir unter dem Integranden nach $\mathrm{x}$ entwickeln dürfen, wenn also $\hat{\phi}^{\prime}(\mathrm{s})$ in der Umgebung von $\mathrm{s}=0$ so stark verschwindet, daß alle Potenzen von 1/s, die bei der Entwicklung auftreten, überkompensiert werden. Das leisten Funktionen vom Typus $\mathrm{e}^{-1 / \mathrm{s}}$ und solche, die in einem endlichen Bereich um die Nullstelle konstant sind. Wir beschränken uns auf den letzten Typ aus einem bald hervortretenden Grund:

Strablungsbedingung: $\hat{\phi}(\mathrm{s})=1$ für $0 \leq \mathrm{s} \leq \mathrm{s}_{0}$.

Die Stabilitätsbedingung folgt ebenfalls aus G1.29. Wir geben ihr folgende hinreichende Gestalt:

Stabilitätsbedingung: $\hat{\Phi}(\mathrm{s}) \geq 0$ für $0 \leq \mathrm{s}<\infty$.

Wenn man zunächst nur die Nullstellen von $M(x)$ ins Auge faßt, erkennt man den hinreichenden Charakter der Bedingung aus Gl. (28). Für reelle $\mathrm{x}$ folgt sofort, daß beide Faktoren im Integranden positiv sind und damit das Integral. Bei rein imaginären $\mathrm{x}=\mathrm{i} \eta$ betrachten wir den Imaginärteil. Er ist nur im Bereich $0 \leq \mathrm{s} \leq \eta$ von Null verschieden und hat darin überall das gleiche Vorzeichen, so daß der Imaginärteil des Integrals und damit auch dieses selbst nicht verschwinden können. Ähnlich können wir für beliebige komplexe $\mathrm{x}=\xi+\mathrm{i} \eta$ argumentieren. Für einen bestimmten Wert von $\mathrm{x}$ ist der Imaginärteil von $\mathrm{s}^{2}+\mathrm{x}^{2}=\mathrm{s}^{2}+\xi^{2}-\eta^{2}+2 \xi \eta$ kon- 
stant. Daraus folgt, daß der Imaginärteil der reziproken Wurzel dieses Ausdrucks zwar nicht mehr konstant ist, aber auf demselben Ri e m a n n schen Blatt überall dasselbe Vorzeichen hat, woraus wiederum unsere Behauptung folgt. Man kann die Bedingung $\mathrm{V}$ auch nach der andern Seite abgrenzen und zeigen: Die Massenstabilität ist sicher nicht vorhanden, wenn

$\mathrm{A}=\int_{0}^{\infty} \hat{\Phi}(\mathrm{s}) \mathrm{s} \mathrm{d} \mathrm{s} \leq 0$ oder $\mathrm{A}>0, \mathrm{~B}=\int_{0}^{\infty} \hat{\phi}(\mathrm{s}) \mathrm{s}^{\mathbf{2}} \mathrm{d} \mathrm{s}<0$

ist. Auf den Beweis wollen wir hier nicht eingehen $^{16}$.

Bisher haben wir nur von den Nullstellen der Funktion M (p) gesprochen und gezeigt, daß Ungleichung (32) zu Ansätzen führt, die jede Nullstelle ausschließen. Massenstabilität herrscht aber nur dann, wenn die Funktion M (p) mindestens in der positiven Halbebene auch keine Verzweigungspunkte hat. Wegen der Beschränktheit der Potentialoberfunktion kann man die Wurzeln in Gl. (28) in der Umgebung eines jeden Punktes $\mathrm{x}$ außerhalb der imaginären Achse unabhängig von $s$ in eine Potenzreihe entwickeln, so daß diese Forderung von selbst erfüllt ist. Verzweigungspunkte sind danach höchstens auf der imaginären Achse möglich. Dort spielen sie eine wesentliche Rolle. Hätte die Funktion M (p) weder Nullstellen noch Verzweigungspunkte, so wäre die durch Gl. (3) bestimmte Funktion unabhängig vom Integrationsweg eindeutig bestimmt und würde im allgemeinen für $t<0$ nicht verschwinden. Stu eckelber $g^{3}$ diskutiert in dieser Hinsicht ein früher von uns angegebenes Beispiel ${ }^{6}$ und erhält eine reine Vorwirkungsfunktion. Im allgemeinen sind Vorwirkungen und Nachwirkungen zugleich zu erwarten. Ein Beispiel, das zufällig nur Nachwirkungen liefert, also ein Gegenbeispiel zu dem erwähnten, ist nicht bekannt. Wir sind darum gezwungen, von mehrdeutigen Ansätzen auszugehen und auf Grund des Kausalitätsprinzips die Nachwirkungsfunktion auszusondern. Die Existenz von Verzweigungspunkten, die eine solche Mehrdeutigkeit garantie-

${ }^{16}$ Wir verzichten an dieser Stelle auf die Wiedergabe weiterer Feinheiten, die den Spielraum zwischen notwendigen und hinreichenden Bedingungen einengen. ren, wird durch die Annahme von Unstetigkeiten in den höheren Ableitungen der Oberfunktion sichergestellt, z. B. durch die spezielle Formulierung der Strahlungsbedingung in Gl. (31), die hierdurch ihre Rechtfertigung findet.

Zum Schluß diskutieren wir kurz einige Beispiele, in ihrer Beziehung zu unsern Bedingungen:

1. Maxwellsche Theorie:

$$
\begin{array}{ll}
\text { Coulomb-Potential } & \Phi(\varrho)=\frac{1}{\varrho}, \\
\text { Oberfunktion } & \hat{\Phi}(\mathrm{s})=1 .
\end{array}
$$

Außer der Endlichkeitsbedingung in Gl. $(25,2)$ sind alle Bedingungen erfüllt.

2. Y ukawa-Theorie des Elektrons $\left(\mathrm{B} \circ \mathrm{pp}^{8}\right)$ :

Statisches Potential $\quad \Phi(\varrho)=\frac{1-\mathrm{e}^{-\varrho}}{\varrho} ;$

Oberfunktion $\hat{\Phi}(\mathrm{s})=1$ für $\mathrm{s} \leq \frac{4}{3},=0$ für $\mathrm{s}>\frac{4}{3}$.

Alle Bedingungen außer der Photonenbedingung in Gl. (27) erfüllt.

\section{Diracsche Theorie des Elektrons :}

Statisches Potential $\Phi(\varrho)=\frac{1}{\varrho}$ für $\varrho>0,=\frac{4}{3}$ für $\varrho=0$;

Oberfunktion $\hat{\phi}(\mathrm{s})=+1$ für $\mathrm{s} \leq \mathrm{s}_{0}$,

$$
\begin{gathered}
=0 \text { für } \mathrm{s} \geq 2 \mathrm{~s}_{0}-\frac{4}{3},=-1 \text { für } \mathrm{s}_{0}<\mathrm{s}_{\mathrm{s}}<2 \mathrm{~s}_{0}-\frac{4}{3} \\
\text { im Limes } \mathrm{s}_{0} \rightarrow \infty .
\end{gathered}
$$

Alle Bedingungen außer der der Massenstabilität erfüllt. Nach Ungleichung (33) ist

$$
\mathrm{A}=-\mathrm{s}_{0}{ }_{0}+\frac{16}{3} \mathrm{~s}_{0}-\frac{16}{3}<0 \text { für } \mathrm{s}_{0}>_{4} \text {. }
$$

4. Ein verträglicher Ansatz $\left(\mathrm{s}_{0}=\frac{4}{3}\right)$ :

Oberfunktion $\hat{\phi}(\mathrm{s})=1$ für $\mathrm{s} \leq \mathrm{s}_{0}-\mathrm{a},=0$ für $\mathrm{s} \geq \mathrm{s}_{0}+\mathrm{a}$, $=\frac{1}{2}-\frac{3}{4} \frac{\mathrm{s}-\mathrm{s}_{0}}{\mathrm{a}}+\frac{1}{4}\left(\frac{\mathrm{s}-\mathrm{s}_{0}}{\mathrm{a}}\right)^{3}$ für $\mathrm{s}_{0}-\mathrm{a}<\mathrm{s}<\mathrm{s}_{0}+\mathrm{a}$.

Im Grenzfall $a \rightarrow 0$ erhält man Beispiel 2. Das statische Potential hat die Form:

$$
\begin{gathered}
\Phi(\varrho)=\frac{1-\mathrm{e}^{-\mathrm{s}_{0} \varrho}}{\varrho}+ \\
+\mathrm{e}^{-\mathrm{s}_{0} \varrho}\left[1+\frac{3}{2 \mathrm{a}} \frac{\mathrm{d}}{\mathrm{d} \varrho}+\frac{1}{2 \mathrm{a}^{2}} \frac{\mathrm{d}^{2}}{\mathrm{~d} \varrho^{2}}\right] \frac{\operatorname{Sin~a} \varrho}{\varrho} .
\end{gathered}
$$

